\title{
EDITORIAL
}

\section{Advances in fracture and damage mechanics}

\author{
M. H. Aliabadi • J. Alfaiate
}

Received: 12 June 2009 / Accepted: 12 June 2009 / Published online: 7 July 2009

(C) Springer Science+Business Media B.V. 2009

This special issue originates from selected contributions to the international conference on Fracture and Damage Mechanics (http://fdm.engineering conferences.net/). The purpose of the meeting was to promote further international co-operation among researchers from different disciplines involved in the study of advanced theoretical, computational and experimental fracture and damage mechanics. The meeting was attended by researchers in academia and industry from over 22 countries presenting 250 papers.

The topics covered include: fatigue, failure analysis, damage mechanics, structural integrity, multiscale modelling, composites, computational methods, experimental measurements and industrial application and processes. The papers presented in this issue consist of a well-balanced mixture of the above covering fundamental concepts as well as practical ones.

We would like to thank Professor Ravi-Chandar for his help and encouragements throughout this project. Special thanks are due to the authors for their patience as the review process took over a year to complete.

M. H. Aliabadi $(\varangle)$

Head, Department of Aeronautics, Imperial College London, South Kensington, London SW7 2BZ, UK

e-mail: m.h.aliabadi@imperial.ac.uk

J. Alfaiate

Instituto Superior Tecnico, Rovisco Pais 1049-001,

Lisbon, Portugal

e-mail: alfaiate@net.sapo.pt 